\title{
Cirugía toracoscópica unipuerto: experiencia en la Fundación Valle del Lili
}

\section{Uniport thoracoscopic surgery: experience at the Fundación Valle del Lili}

Mauricio Velásquez Galvis ${ }^{(1)}$; Andrés Fernando Dominguez Calero(2)

RESUMEN

La forma de realizar la cirugía toracoscópica ha venido presentando cambios y ha evolucionado en la medida en que el desarrollo tecnológico ha desarrollado nuevos instrumentos que permiten tener mayor precisión y seguridad. En la actualidad se pueden encontrar algunas experiencias con diferentes procedimientos torácicos realizados por un solo puerto. Se diseñó un trabajo con el objetivo de evaluar los desenlaces de los pacientes llevados a cirugía torácica vídeo-asistida a través de un solo puerto.

Se realizó un trabajo descriptivo, prospectivo, en el que se incluyeron todos los pacientes que fueran llevados a algún procedimiento de cirugía de tórax planeado para realizarse por toracoscopia y que hubiera sido hecho por un solo puerto, estudiando variables demográficas, tiempo operatorio, estancia hospitalaria, duración del tubo de tórax y complicaciones relacionadas con el procedimiento.

Se operaron 38 pacientes; la mayoría de los procedimientos efectuados fueron pleurodesis con talco para derrames malignos y decorticaciones pulmonares por empiemas pleurales. La mediana de tiempo quirúrgico fue de 42 minutos y la estancia hospitalaria fue de ocho dias. Las complicaciones más frecuentes fueron respiratorias, como la necesidad de uso de ventilación mecánica no invasiva. Falleció un paciente debido a su enfermedad avanzada.

La cirugía torácica video-asistida a través de un sólo puerto representa una evolución de la cirugía toracoscópica tradicional. Es un procedimiento simple, efectivo y beneficioso para los pacientes, con una morbilidad minima.

Palabras clave: toracoscopia, unipuerto.

\section{ABSTRACT}

Thoracoscopic surgical techniques have changed and evolved as technological development has produced instruments capable of greater precision and safety. Today it is possible to find some experiences with different thoracic procedures performed through a single incision. A study was designed with the aim of assessing the outcomes of patients undergoing video-assisted thoracic surgery performed through a single incision.

A descriptive, prospective study was carried out. The study included all patients who underwent surgery performed by thoracoscopy through a single port. The study assessed demographic variables, duration of surgery, length of hospital stay, time with chest tube, and complications related with the procedure.

Thirty eight (38) patients were operated. The most common procedures were talc pleurodesis for malignant pleural effusions and pulmonary decortication for empyema. The median surgical time was 42 minutes, and the median hospital stay was 8 days. The most common complications were of respiratory origin, such as the need for non-invasive mechanical ventilation. Only one patient died, due to advanced illness.

Video-assisted thoracic surgery performed through a single incision is an evolution of traditional thoracoscopic surgery. It is a simple, effective, and beneficial procedure, whose morbidity is minimal.

Keywords: thoracoscopy, uniportal surgery.

Rev Colomb Neumol 2013; 24 (2):80-83

(1) Cirujano de Tórax, Fundación Valle del Lili, Cali, Colombia.

(2) Residente II año, Universidad CES, Fundación Valle del Lili, Cali, Colombia.

Correspondencia: Doctor Mauricio Velásquez Galvis, Correo electrónico: mauriciovelasq@yahoo.com

Recibido: septiembre 13 de 2012. Aceptado: noviembre 22 de 2012. 


\section{INTRODUCCIÓN}

La cirugía torácica video-asistida ha cambiado definitivamente el manejo quirúrgico y la evolución postoperatoria de los pacientes con diferentes patologias pulmonares y mediastinales.

La forma de realizar la cirugia toracoscópica también ha sufrido cambios y ha evolucionado en la medida en que el desarrollo tecnológico ha creado nuevos instrumentos que permiten tener mayor precisión y seguridad, de tal manera que la forma en que tradicionalmente se enseñaba a ubicar los puertos para acceder a la cavidad torácica ha venido adaptándose a las necesidades de cada paciente y a la experiencia de cada cirujano. En la actualidad se pueden encontrar algunas experiencias con diferentes procedimientos torácicos realizados por un solo puerto $(1,2)$.

En este trabajo se presenta la experiencia de un centro en la realización de cirugía toracoscópica por un solo puerto.

\section{MATERIALES Y MÉTODOS}

Durante el período de tiempo comprendido entre enero de 2010 y diciembre de 2010 se diseñó un estudio descriptivo, prospectivo, con el objetivo de evaluar los desenlaces de los pacientes llevados a cirugia torácica video-asistida a través de un solo puerto, en el que se incluyeron todos los pacientes mayores de 18 años que fueran llevados a algún procedimiento de cirugia de tórax planeado para realizarse por toracoscopia y que hubiera sido hecho por un solo puerto.

Se excluyeron los pacientes en quienes, por cualquier razón durante la cirugía, se hubiera tenido que fabricar otro puerto, asi como los pacientes que se hubieran tenido que convertir a cirugia abierta.

Se estudiaron variables demográficas y otras de interés como tiempo operatorio, estancia hospitalaria, duración del tubo de tórax y complicaciones relacionadas con el procedimiento o con la enfermedad de base.

La técnica empleada es similar a la descrita por Rocco (3) y comprende una intubación selectiva con tubo bilumen o una técnica con anestesia peridural y con el paciente despierto, lo cual fue libremente escogido por el grupo de anestesia; luego se ubicaba al paciente en decúbito lateral y se realizaba una única incisión, estandarizada en el quinto espacio intercostal entre las lineas axilar anterior y media, de unos 2 a 4 centímetros y a través de la cual se introducía un puerto para la cámara, que además permitia la introducción del instrumental necesario para cada tipo de cirugia. Una vez finalizado el procedimiento se dejaba un tubo de tórax \#28 que era fijado con un material de sutura no absorbible; posteriormente se cerraban un plano muscular y otro subcutáneo con un material absorbible y finalmente la piel.

Para la recolección de los datos se diseñó un instrumento en el que se mantenia la confidencialidad de los pacientes, por lo que no se consideró necesario firmar un consentimiento informado. Los datos se procesaban en Excel de Microsoft, con el cual se hizo el análisis estadístico utilizando las medidas de normalidad expresadas en medianas y rangos intercuartílicos.

\section{RESULTADOS}

Se operaron 38 pacientes, 19 mujeres $(50 \%)$ y 19 hombres $(50 \%)$. Los datos demográficos se observan en la tabla 1. La mayoria de los procedimientos realizados fueron pleurodesis con talco para derrames malignos y decorticaciones pulmonares por empiemas pleurales.

En la tabla 2 se muestran los resultados de las variables operatorias.

Las complicaciones encontradas se registran en la tabla 3; de éstas las más frecuentes fueron respiratorias, como requerimiento de ventilación mecánica,

Tabla 1. Datos demográficos.

\begin{tabular}{|llr|}
\hline Edad (años) & & $60(46-74)^{*}$ \\
Diagnóstico & & $\mathrm{n}(\%)$ \\
& Derrame pleural maligno & $13(34 \%)$ \\
& Derrame pleural & $2(5 \%)$ \\
& Empiema & $10(26 \%)$ \\
& Nódulo pulmonar & $7(18 \%)$ \\
& Neumotórax & $3(8 \%)$ \\
& Malformación arteriove- & $1(3 \%)$ \\
& nosa pulmonar & \\
& Cáncer de pulmón & $1(3 \%)$ \\
& Tumor fibroso de pleura & $1(3 \%)$ \\
Procedimientos & & \\
& Pleurodesis con talco & $12(33 \%)$ \\
& Resección pulmonar en & $10(26 \%)$ \\
& cuña & \\
& Decorticación pulmonar & $13(34 \%)$ \\
& Lobectomía pulmonar & $3(8 \%)$ \\
\hline
\end{tabular}

* Valores en mediana y rango intercuartilico. 
Tabla 2. Resultados postoperatorios.

\begin{tabular}{|lc|}
\hline Variable & Mediana (riq) \\
\hline Tiempo quirúrgico (minutos) & $43(30-60)$ \\
Tubo de tórax (días) & $4(3-6)$ \\
Estancia hospitalaria (días) & $8(6-60)$ \\
\hline
\end{tabular}

Tabla 3. Complicaciones postoperatorias.

\begin{tabular}{|ll|}
\hline Complicación & $\mathbf{n}$ \\
\hline VM & 8 \\
VMNI & 9 \\
Traqueostomía & 2 \\
Complicaciones respiratorias & 3 \\
Hipotensión & 4 \\
Uso de vasoactivos & 3 \\
Complicaciones cardiacas & 1 \\
\hline
\end{tabular}

VM: ventilación mecánica.

VMNI: ventilación mecánica no invasiva.

ventilación mecánica no invasiva y presencia de neumonía (dos pacientes) y de atelectasia (un paciente). Sólo se registró una complicación cardiaca en un paciente que tuvo fibrilación auricular y que cedió con manejo médico.

Sólo un paciente falleció debido a un cáncer de esófago avanzado que presentó complicaciones sépticas por perforación de la masa tumoral.

\section{DISCUSIÓN}

La cirugia toracoscópica ha cambiado la forma en que se manejan los pacientes de cirugía de tórax; de igual manera, este tipo de cirugía ha venido evolucionando y cada vez se disminuyen los puertos de abordaje para la realización de los procedimientos pulmonares y mediastinales. Desde 2004 aparece la primera descripción de resecciones pulmonares en cuña para el diagnóstico de enfermedades intersticiales realizadas a través de un solo puerto con excelentes resultados en cuanto a estancia hospitalaria, duración del tubo de tórax y dolor postoperatorio $(4,5)$.

En este trabajo se encontró que el abordaje unipuerto para los procedimientos de cirugía torácica es factible y permite efectuar diferentes cirugias, algunas simples, como la pleurodesis con talco, hasta procedimientos tan complejos como la lobectomía pulmonar total con vaciamiento ganglionar por cáncer de pulmón.

Rocco y colaboradores habian mostrado una duración del tubo de tórax y una estancia hospitalaria de
$1(1-6)$ y $2,5(1-6)$ dias respectivamente (6), lo cual es menor si se compara con nuestros resultados. Sin embargo, en el trabajo de Rocco sólo se hicieron cuñas pulmonares para enfermedades intersticiales o neumotórax, mientras que en este reporte se incluyen otros tipos de diagnósticos y cirugias que pueden aumentar un poco más la estancia hospitalaria y la duración del tubo de tórax. Lo anterior se puede observar mejor en el trabajo de Migliore (7), en el que la estancia hospitalaria fue de 6 a 8 dias según la patología (benigna o maligna), y la duración del tubo de tórax fue de 5 dias, resultados comparables con los nuestros.

Los tiempos quirúrgicos son similares en todos los trabajos $(4,6,7)$ y oscilan en los 65 minutos; no obstante, encontramos que la mediana del tiempo operatorio fue de 42 minutos.

Las complicaciones reportadas más frecuentes son las respiratorias debido más a las enfermedades de base que a los procedimientos realizados. Migliore (7) reporta una morbilidad en 19 pacientes, mas no especifica en qué consistió, mientras que en nuestro trabajo se reporta como la principal complicación el uso de ventilación mecánica invasiva y no invasiva en ocho y nueve pacientes, respectivamente (Tabla 3 ).

En cuanto a las tres lobectomías, que merecen comentario aparte, sólo podrian compararse con el reporte de González y colaboradores (8) en el que se hizo igualmente una lobectomía pulmonar inferior izquierda con un tiempo quirúrgico de 110 minutos y una estancia hospitalaria de tres días, hecho comparable con nuestros datos. De igual forma, se encuentran resultados muy similares en los tres pacientes, excepto en uno que tuvo una estancia de ocho dias debido a neumotórax residual luego del retiro del tubo de tórax y que finalmente tuvo desenlace sin otros contratiempos.

\section{CONCLUSIONES}

La cirugía torácica video-asistida a través de un solo puerto, representa una evolución de la cirugía toracoscópica tradicional con unos resultados comparables. Es un procedimiento simple, efectivo y beneficioso para los pacientes, cuya morbilidad es mínima. Se requieren más estudios que permitan comparar los desenlaces de los procedimientos realizados por un solo puerto frente a los que utilicen más incisiones.

\section{BIBLIOGRAFÍA}

1. Salati M, Brunelli A, Rocco G. Uniportal video-assisted thoracic surgery for diagnosis and treatment of intrathoracic conditions. Thorac Surg Clin. 2008; 18: 305-310. 
2. Rocco G, Romano V, Accardo R, Tempesta A, La Manna C, La Rocca A, e al. Awake single-access (Uniportal) video-assisted thoracoscopic surgery for peripheral pulmonary nodules in a complete ambulatory setting. Ann Thorac Surg. 2010; 89: 16251627.

3. Rocco G. Single-port video-assisted thoracic surgery (uniportal) in the routine general thoracic surgical practice. Op Tech Thorac Cardiovasc Surg. 2009; 14: 326-335.

4. Rocco G, Martin-Ucar A, Passera E. Uniportal VATS wedge pulmonary resections. Ann Thorac Surg. 2004; 77: 726-728.

5. Jutley R, Khalil M, Rocco G. Uniportal versus standar three-port VATS technique for espontaneous pneumothorax: Comparison of post-operative pain and residual paraesthesia. Eur J Cardiothorac Surg. 2005; 28: 43-46.

6. Rocco G, Khalil M, Jutley R. Uniportal video-assisted thoracoscopic surgery wedge lung biopsy in the diagnosis of interstitial lung diseases. J Thorac Cardiovasc Surg. 2005; 129: 947-948.

7. Migliore M. Efficacy and safety of single-trocar technique for minimally invasive surgery of the chest in the treatment of noncomplex pleural disease. J Thorac Cardiovasc Surg. 2003; 126 : 1618-1623.

8. González D, Delgado M, Paradela M, Fernández R. Uni-incisional video-assisted thoracoscopic left lower lobectomy in a patient with an incomplete fissure. Innovations 2011; 6: 45-47. 